\title{
Performance Evaluation and Optimization of B.A.T.M.A.N. V Routing for Aerial and Ground-based Mobile Ad-hoc Networks
}

\author{
Benjamin Sliwa, Stefan Falten and Christian Wietfeld \\ Communication Networks Institute, TU Dortmund University, 44227 Dortmund, Germany \\ e-mail: $\{$ Benjamin.Sliwa, Stefan.Falten, Christian.Wietfeld\}@tu-dortmund.de
}

\begin{abstract}
The provision of reliable and efficient end-toend communication within ground- and air-based mobile mesh networks is a major challenge for routing protocols due to the mobility-related dynamics of the channel properties and the resulting mesh network topology. In this paper, we evaluate the performance of the novel Better Approach To Mobile Adhoc Networking (B.A.T.M.A.N.) V routing protocol for vehicular mesh networks and propose a mobility-predictive extension that explicitly addresses highly dynamic communication networks. In order to enable large-scale simulative analysis, we present an open source simulation model, which is validated by field experiments. Within a comprehensive evaluation campaign in Vehicle-to-Everything (V2X) and Unmanned Aerial Vehicle (UAV) scenarios, it is shown that the predictive B.A.T.M.A.N. V-based approach is significantly better suited for maintaining reliable connectivity within highly mobile mesh networks than established routing protocols.
\end{abstract}

\section{INTRODUCTION}

The usage of multi-hop communication within mesh-based Mobile Ad-hoc Networks (MANETs) enables significant range extensions as well as path redundancy, which is exploited in vehicular platooning, remote sensing of disaster areas using UAVs and ad-hoc network provisioning (c.f. Fig. 11). Due to the potentially high relative mobility of the different nodes and the short lifetime of routing paths, which results in a highly dynamic network topology, routing is immanently challenging in these networks.

A promising solution approach that explicitly addresses these challenges, is the usage of anticipatory communication [1], [2], which aims to utilize the existing communication network in a more efficient way by an increased situationawareness. Cross-layer principles allow the integration of the mobility characteristics of the vehicles themselves into the routing decisions.

In this paper, we extend previous work on mobility-aware vehicular mesh routing [3] based on the B.A.T.M.A.N. routing protocol [4] in order to leverage new features of the fifth protocol version. Unlike most established routing protocols, which handle routing on the network layer, B.A.T.M.A.N. operates on the Medium Access Control (MAC) layer since version IV [5]. Moreover, version V introduces major changes and novelties in the routing behavior and its metrics. In the remainder of this paper, the following contributions to anticipatory mesh networking are provided:
- Presentation of a novel open source simulation model of B.A.T.M.A.N. V for Objective Modular Network Testbed in $\mathrm{C}++(\mathrm{OMNeT}++)$ [6] and its INET/ INETMANET framework, which is close to the Linux kernel implementation of the protocol.

- Simulation model validation using field measurements and empirical channel modeling in Sec. V

- Optimization and extension of B.A.T.M.A.N. V for high mobility scenarios using mobility prediction in Sec. III

- Performance comparison of B.A.T.M.A.N. V and the proposed experimental extensions with established protocols in aerial and ground-based simulation scenarios in Sec. VI.

- All raw results are provided in an open access way.

\section{RELATED WORK}

In this section, we discuss current approaches for vehicular mesh routing and provide information about the general properties of the considered B.A.T.M.A.N. protocol.

\section{A. Overview of Current Vehicular Mesh Routing Approaches}

A detailed overview about current Vehicular Ad-hoc Network (VANET) - which form a subclass of MANETs- trends and research topics based on literature analysis is provided by [7]. While routing is pointed out as one of the major VANET research topics, it is stated that traditional (topology-based) routing protocols mostly cannot satisfy the requirements of highly mobile vehicular scenarios. Similarly, the authors of [8] emphasize the need for cross-layer design techniques in order to increase the situation-awareness for routing in mobile networks. Geo-based approaches such as Greedy Perimeter Stateless Routing (GPSR) [9] exploit positioning information for routing decisions, which has been demonstrated to work efficiently in vehicular networks. Consequently, predictive extensions have been proposed, which go another step further by integrating locations forecasts into the routing process for estimating link stability times [10], [11]. Those approaches are usually implemented as an extension to existing protocols e.g., based on Ad hoc On-demand Distance Vector (AODV) [12] in [13] and GPSR-based in [14]. However, although existing solutions achieve significant improvements in the resulting transmission efficiency, the approaches are mostly 
generic and rely on naive predictions, e.g., extrapolations based on position, direction and velocity.

Although in the past the research in vehicular and aerial mesh networks has mostly been carried out with an isolated view on the intended usecase, recent works emphasize their similarities [15] and advocate for a joint consideration of these vehicle types within upcoming Intelligent Transportation System (ITS) scenarios, e.g., near-field delivery and ad-hoc network provisioning and sensing at road incidents by UAVs.

\section{B. General Properties of the B.A.T.M.A.N. Routing Protocol}

B.A.T.M.A.N. [12] has been proposed by the Freifunk community after the application of Optimized Link State Routing (OLSR) [16] did not fulfill the performance requirements of large-scale mesh deployments. Although B.A.T.M.A.N. is a proactive protocol and shows a similar performance as other established protocols, it has some unique properties that arise from its bio-inspired nature, which implement the idea of stigmergy.

- B.A.T.M.A.N. strictly follows a decentralized routing approach: Instead of requiring complete knowledge about the network topology, only information about the suitability of the direct neighbors for reaching a defined destination is maintained, the network in between is considered a black box. As information is redundantly received from all other nodes of the network - which are referred to as originators - via multiple paths through the immediate neighboring nodes, it is highly robust against packet loss.

- The periodically transmitted Originator Messages (OGMs), which contain information about the reverse path quality, are propagated through the whole network. The metric calculation and message handling can easily be exchanged by another method, making it a promising research platform for optimization and development of new link quality assessment methods.

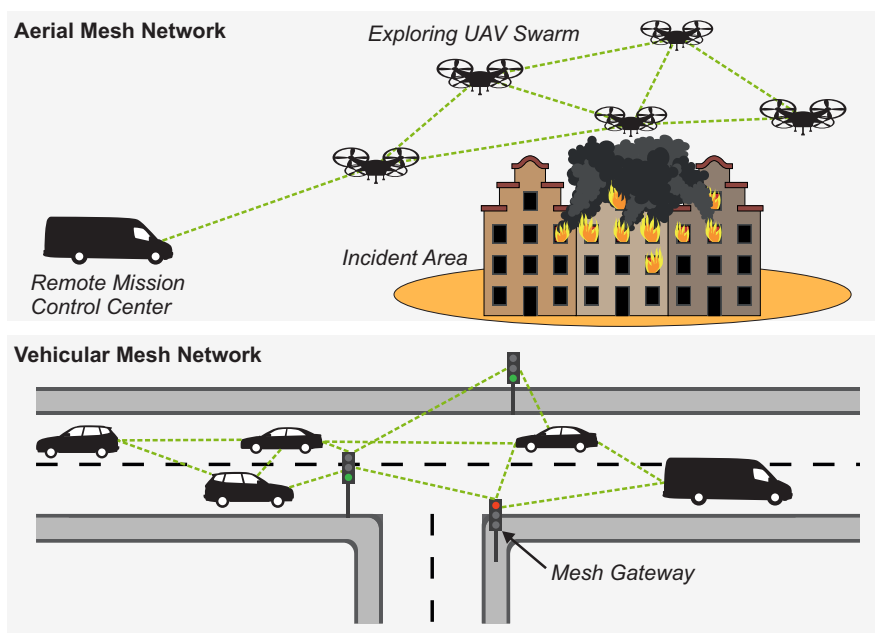

Fig. 1. Example applications for aerial and vehicular mesh networks.

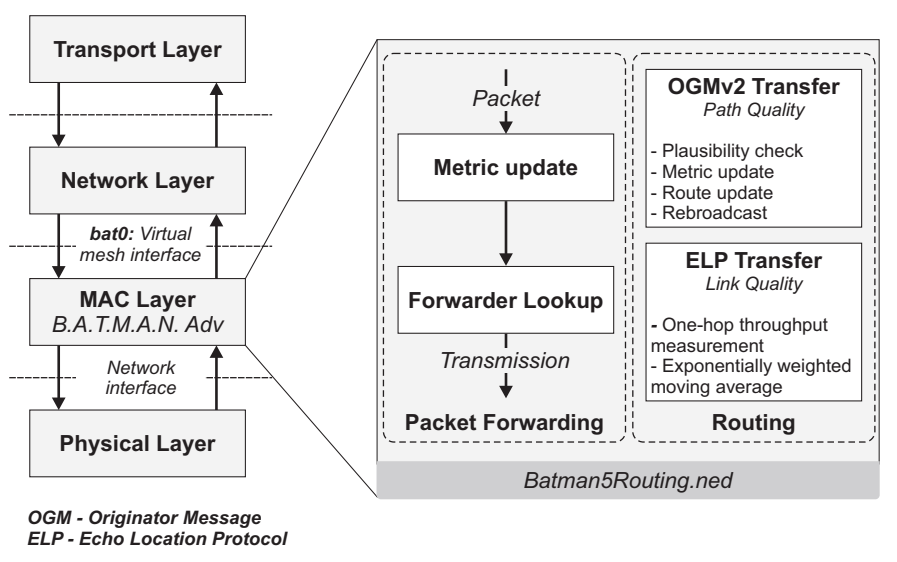

Fig. 2. Architecture model and message flow for B.A.T.M.A.N. V routing.

- Since multiple paths are maintained for each destination, multi-path communication can be integrated for load balancing [17].

- In contrast to other protocols, which are often only evaluated in simulators, the development of B.A.T.M.A.N. V is driven by a practical usecase intention and implementations are available for Linux-based operating systems.

\section{Simulation-BASEd System Model}

In this section, the simulation model and its extensions for highly mobile scenarios are presented.

An illustration of the overall architecture model and the message flow is shown in Fig. 2 As an entry point for all exchanged packets, the mesh interface bat 0 is used to abstract the underlying mesh topology of the network, acting as a virtual network switch. All routing messages are encapsulated in raw Ethernet frames. The MAC layer approach allows the implementation of the routing mechanism as a linux kernel module, which - in contrast to network layer routing - avoids the necessity for packet exchange between userspace and kernelspace, reducing computing effort and energy consumption.

Although the INETMANET framework of OMNeT++ provides ManetRoutingBase.ned as a common abstraction for MANET protocols, it only considers network layer routing. Therefore, the simulation model is based on a novel logical host module Batman5Router.ned, which links the interfaces of the different logical layers.

The routing protocol uses different packet types to determine the reverse path metric to the originators within the network, which is further explained in the following paragraphs.

\section{A. Link Quality Assessment}

Due to the decentralized approach of B.A.T.M.A.N. V, all decision processes are based on link-level knowledge, which is obtained by periodical exchange of Echo Location Protocol (ELP) messages. In regular protocol implementation, the latter are used to estimate the throughput to the direct neighbors. Additionally, ELP probing triggers the transmission of two unicast ELP messages to each forwarder within the defined 
ELP interval in order to artificially generate traffic for the throughput estimation. While this procedure is mandatory in the linux kernel implementation, it is optional in the simulation model and can be configured to use variable packet sizes, which are further evaluated in Sec. VI As an alternative to the throughput metric, a hopcount-based approach, which uses hop penalties, can be applied. After node $N$ has determined new immediate link metric value $\Phi_{F}^{N}$ to forwarder $F$, the final value is calculated based on the link quality history using Exponentially Weighted Moving Average (EWMA).

\section{B. Routing Process and Metrics}

Each node $N$ maintains a link metric $\Phi_{F}^{N}$ for each of its 1-hop neighbors, based on knowledge obtained from ELP message exchange. Routing decisions are based on the end-toend path metric towards each originator, which is derived from the metrics of the reverse path obtained from the OGMs. Upon creation of an OGM, each originator initializes the reverse path score as the uint 32 maximum $2^{32}-1$ and announces it with the message. The intermediate nodes update the contained value based on their local knowledge about the link quality to the last forwarder. In the proposed simulation model, the metric calculation is formulated in an abstract way in order to emphasize its platform character, which enables the integration of further metrics in the future. As all logical calculations are performed in the continuous domain in the range $[0,1]$, the uint 32 values are transformed into this value range for the processing step and back before the packet forwarding.

Upon reception of an OGM of node $D$ via a forwarder $F$, node $N$ computes the reverse path metric $\Psi_{D \mid F}^{N}$ as

$$
\Psi_{D \mid F}^{N}=\Theta\left(\hat{\Psi}_{D}, \Phi_{F}^{N}\right)
$$

by applying an operator $\Theta$ to the received reverse path metric $\hat{\Psi}_{D}$ and the link metric $\Phi_{F}^{N}$ to the forwarder. A summary of the considered metrics for the different B.A.T.M.A.N. protocol versions and the proposed extensions is given in Tab. I

TABLE I

ESTABLISHED AND EXPERIMENTAL ROUTING METRICS FOR THE DIFFERENT B.A.T.M.A.N. VERSIONS

\begin{tabular}{ll}
\hline Version & Metric \\
\hline III & Average number of received OGMs within a defined \\
& interval \\
IV & Determination of the Transmission Quality (TQ) \\
V & Throughput estimation based on ELP transfer \\
V & Hop count \\
\hline V & Distance-based forwarding using position information \\
V & Mobility prediction \\
\hline
\end{tabular}

1) Distance-based Forwarding: As discussed in II-A, the usage of geo-information is a promising approach for routing in vehicular networks. Therefore, the basic B.A.T.M.A.N. V protocol is extended for geo-based decision making through a novel routing metric, which is based on position announcements within the ELP messages.
With $d_{F}^{N}(t)$ being the distance from node $N$ to node $F$ and $d_{\max }$ being an estimation for the transmission range, the proposed metric update procedure is handled as

$$
\Psi_{D \mid F}^{N}(t)=\hat{\Psi}_{D}-\left(\frac{d_{F}^{N}(t)}{d_{\max }}\right)^{\alpha}
$$

with $\alpha$ as a weighting exponent.

2) Mobility-predictive Forwarding: In order to achieve a higher level of awareness about the future network topology, the geo-based forwarding scheme is extended by a predictive component. Upon transmission of an ELP message, each node predicts its own future position based on trajectory knowledge and announces it within the message.

In the following, it is assumed that each vehicle is able to determine an estimation of its near future trajectory. For completeness, we refer to the analysis in [18], where the accuracy of different mobility prediction approaches is compared in real world vehicular scenarios. The future position $\tilde{\mathbf{P}}(t+\tau)$ is then obtained by virtually moving the vehicle along the path of its trajectory for the duration of $\tau$, which is further described in [18]. The mobility-predictive metric is then calculated as

$$
\Psi_{D \mid F}^{N}(t)=\hat{\Psi}_{D}-\max \left[\left(\frac{d_{F}^{N}(t)}{d_{\max }}\right)^{\alpha},\left(\frac{\tilde{d}_{F}^{N}(t+\tau)}{d_{\max }}\right)^{\alpha}\right]
$$

with $\tau$ being the desired prediction lookahead and $\tilde{d}_{F}^{N}(t+\tau)$ being the anticipated distance between $N$ and $F$.

\section{Methodology}

In this section, the setups for the simulative evaluation and the experimental validation are presented.

\section{A. Evaluation Scenario}

The simulation model is based on OMNeT++ and the INET/INETMANET framework and is provided in an open source way at [19] alongside with the raw results of the performed evaluations. For the field test validation, common of-the-shelf embedded computers are used that run a linuxbased operating system and execute the B.A.T.M.A.N. V (version 2018.0) routing mechanism.

The default parameters for the different scenarios and routing protocols are summarized in Tab. II. For the mobilitypredictive metric, $d_{\max }$ is calculated as the maximum distance that satisfies the receiver sensitivity based on the channel model. The Friis model is used for rural environments and the Nakagami model is used to model urban scenarios. The prediction lookahead is chosen based on the analysis of [3]. For the evaluations, the performance of a multi-hop User Datagram Protocol (UDP) data Constant Bitrate (CBR) stream is analyzed.

\section{B. Mobility Models}

For the simulative performance evaluation, a mixture of generic and vehicle-specific mobility models is applied. The initial performance evaluation and parameter optimization is performed using a Random Waypoint model in order to allow comparisons with other research works, however it is 
TABLE II

Default Simulation Parameters

\begin{tabular}{ll}
\hline Parameter & Value \\
\hline MAC & IEEE 802.11g \\
Channel model: Rural / Urban & $\{$ Friis, Nakagami (m=2) $\}$ \\
Path loss exponent $\eta$ & 2.65 \\
Receiver sensitivity & $-83 \mathrm{dBm}$ \\
Transmission power & $\{10,20\} \mathrm{dBm}$ \\
Carrier frequency & $2.4 \mathrm{GHz}$ \\
Number of mesh nodes & 10 \\
Stream data rate & $10 \mathrm{MBit} / \mathrm{s}$ \\
Simulation duration per run & $300 \mathrm{~s}$ \\
Simulation runs per setting & 25 \\
Playground size: Generic scenario & $600 \mathrm{~m} \times 600 \mathrm{~m} \mathrm{x} \mathrm{10} \mathrm{m}$ \\
Playground size: UAV scenario & $500 \mathrm{~m} \times 500 \mathrm{~m} \times 250 \mathrm{~m}$ \\
Playground size: Vehicular scenario & $1500 \mathrm{~m} \times 1000 \mathrm{~m} \mathrm{x} \mathrm{10} \mathrm{m}$ \\
\hline B.A.T.M.A.N. version & 2018.0 \\
OMNeT++/ INET version & $5.2 .1 / 3.6 .3$ \\
OGM interval & $0.33 \mathrm{~s}$ \\
ELP interval & $0.2 \mathrm{~s}$ \\
Weighting exponent $\alpha$ : Rural / Urban & $\{1,2\}$ \\
Prediction lookahead $\tau:$ Rural / Urban & $\{3,4\} \mathrm{s}$ \\
\hline
\end{tabular}

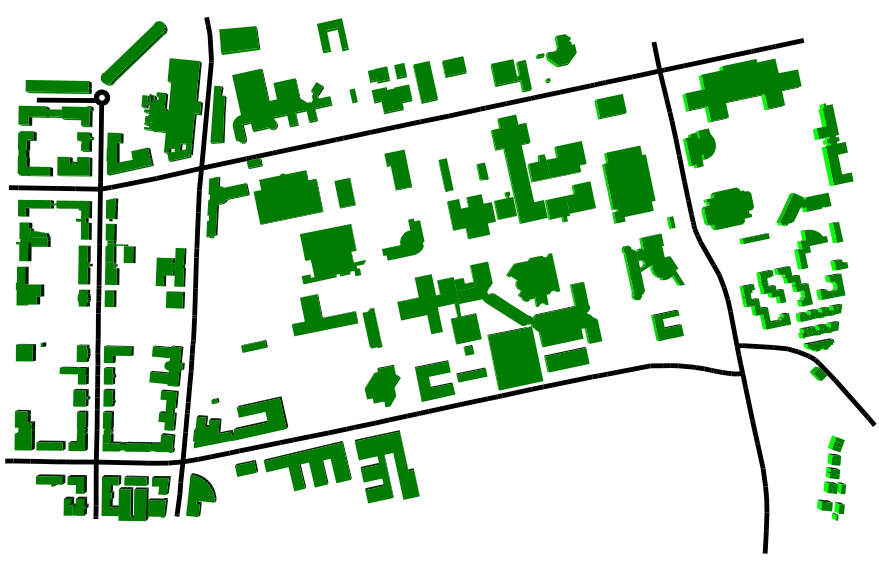

Fig. 3. Map of the campus area for the vehicular evaluation scenario. (Map data: (C) OpenStreetMap contributors, CC BY-SA.)

assumed that vehicles are aware of their future trajectory. For the vehicular scenario, a network provisioning scenario using gateway vehicles is modeled using Lightweight ICTcentric Mobility Simulation (LIMoSim) [20]. 30 cars move around the campus area of the TU Dortmund University, which is illustrated in Fig. 3. One randomly selected vehicle aims to establish a stream data connection to a network gateway vehicle using the mesh for packet forwarding.

For the evaluation of the applicability of the proposed mobility-predictive routing protocol, for low-altitude UAV applications, the Distributed Dispersion Detection (DDD) [21] algorithm applied, which is intended for agent-based plume exploration in hazardous environments. The mesh communication is used to stream video data to a remote mission control center. Here, a trace-based mobility modeling approach is applied based on traces of the MATLAB-based UAV simulation framework of [21]. The UAVs make use of controlled mobility, thus each UAV is aware of its desired trajectory in the near

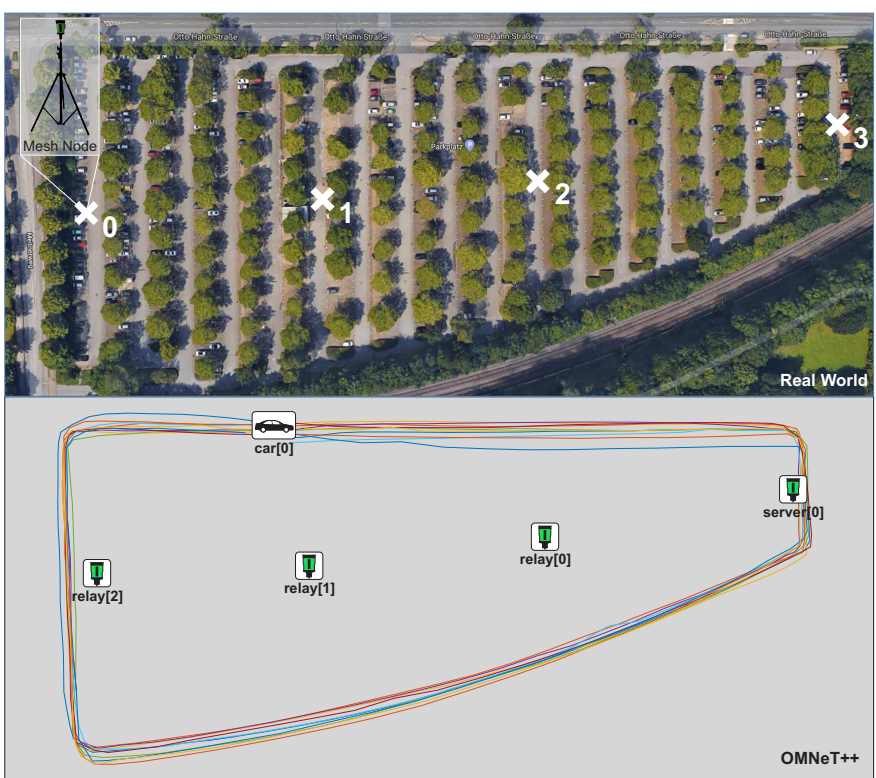

Fig. 4. Overview of the field test scenario and its representation within the OMNeT++ simulator for the validation.

future.

\section{VALIDATION}

In this section, the derived simulation model for B.A.T.M.A.N. $\mathrm{V}$ is validated using field measurements in controlled scenarios using the default routing metric. Within the real world measurements, a mobile vehicle transmits UDP stream data with a traffic load of $10 \mathrm{MBit} / \mathrm{s}$ to a static server node using the mesh network infrastructure. iperf is used for traffic generation and Key Performance Indicator (KPI) measurement. The same scenario is modeled within a simulation setup.

Fig. 4 shows a map of the real world evaluation scenario as well as its representation within the $\mathrm{OMNeT++}$ simulator. All B.A.T.M.A.N. V nodes are mounted on a tripod at $1.5 \mathrm{~m}$ height. For the One-hop scenario, the server is placed centrally between the location markers 1 and 2. Two-hop uses location 2 for the server and location 1 for a single relay node. Multi-hop applies the topology illustrated for the OMNeT++ scenario as shown in Fig. 4. Fig. 5 shows the measured channel properties and the Friis and Two Ray channel models for comparison. It can be seen that the mean attenuation behavior above $70 \mathrm{~m}$ can be approximated by the Friis model with a path loss exponent $\eta$ of 2.65. The Two Ray model does not fit well in the considered small-scale scenario.

In order to achieve a high congruency of the real world and the simulation scenario, an empirical channel model is created based on the field measurements and used for the following validation. The resulting data rate and delay of the measurement campaign and its simulation representation is shown in Fig. 6. The results substantiate the outcome of the channel behavior analysis. In the considered small-scale range, 
the Two Ray model is mostly in the constructive interference region with an unrealistic high data rate, which is more than twice as high as the measurement, therefore it is not considered further. The Nakagami model provides a close approximation of the real world behavior of the data rate but fails to model the delay behavior with a sufficient accuracy. It can been seen that the best match for both indicators is achieved by the empirical channel model that is based on the measured channel characteristics. In conclusion, the proposed simulation model for B.A.T.M.A.N. V and its real world implementation show similar average characteristics and results.

\section{Vi. Simulative Performance Evaluation}

In this section, B.A.T.M.A.N. $\mathrm{V}$ and its proposed extensions are evaluated in different scenarios and compared with the established routing protocols AODV [12], OLSR [16], and B.A.T.M.A.N. III [4]. All errorbars show the 0.95-confidence interval of the mean value. At first, the parametrization of B.A.T.M.A.N. V is optimized for the mobile scenario based on random waypoint mobility. Afterwards, the performance of the protocol is evaluated in two reference scenarios focusing on vehicular and UAV mobility.

\section{A. Optimization for Highly Mobile Scenarios}

B.A.T.M.A.N. V introduces ELP probing, which is intended to provide the throughput metric with further traffic for the link quality assessment. Therefore, the effects of the additional messages are evaluated. Fig. 7 shows the impact of ELP probing on the resulting data rate for different ELP packet sizes. It can be seen that the amount of additional messages has a negative impact on the routing performance in the mobile scenario due to the increased amount of collisions. Therefore, ELP probing is disabled for the following evaluations.

A comparison of the native throughput metric with the proposed mobility-aware extensions is shown in Fig. 8. Although both proposals outperform the native approach, the general Packet Delivery Ratio (PDR) in the rural scenario is very high, thus limiting the optimization potential. In the urban scenario, the throughput-based approach suffers from paket loss. Here, the mobility-aware approaches are able to achieve significant benefits, with the predictive approach increasing the PDR by $23 \%$. Therefore, only the proposed predictive B.A.T.M.A.N. $\mathrm{V}$ metric will be considered in the further evaluations.

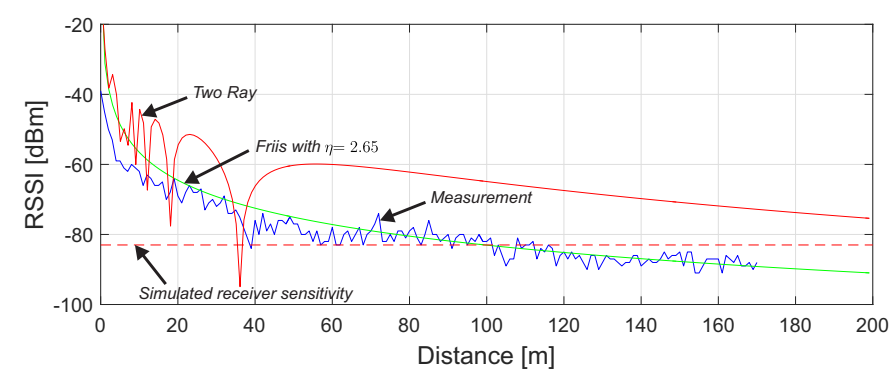

Fig. 5. Empirically measured channel properties and channel model approximation.
Fig. 9 shows a scalability analysis with varied vehicle speed, traffic load and number of streams. In general, the extended B.A.T.M.A.N. $\mathrm{V}$ and reactive AODV achieve significantly higher PDR values than OLSR and B.A.T.M.A.N. III. Due to its mobility-related metric, the proposed extended B.A.T.M.A.N. $\mathrm{V}$ has a higher dependency to the velocity than the topology-based protocols, resulting in a steeper PDR decrease with increased speed. With the traffic load of the single stream being varied, the PDR is only slightly reduced for all considered protocols. Furthermore, the behavior of the protocol for a varied number of $2 \mathrm{MBit} / \mathrm{s}$ streams with random source-destination pairs is analyzed. Here, the extended B.A.T.M.A.N. V behaves slightly better than AODV. Up to three parallel streams, AODV benefits from the increased traffic on multiple end-to-end paths. Although AODV is a reactive protocol, all intermediate nodes of an active path maintain the reverse path quality for a defined time period. Therefore, the presence of multiple active paths increases the probability that the nodes are able to switch the routing path within a changed network topology without requiring a route request phase. For a higher number of active streams, the increasing loss probability for routing messages significantly decreases the routing performance for all considered protocols.

\section{B. UAV Evaluation Scenario}

Fig. 10 shows the resulting PDR and delay values for the considered protocols in the UAV-based plume exploration scenario using DDD. As the mobility algorithm itself is connectivity-aware, it aims to maintain the swarm coherence by adjusting the mobility behavior of the UAVs, which results in a generally high PDR level. Although the mean PDR value of the proposed approach is only slightly better than the result of OLSR and B.A.T.M.A.N. III, the occurrence of lower PDR values is significantly reduced. While all proactive protocols achieve a similar delay, the latter is significantly higher for AODV due to its on-demand approach.

\section{Vehicular Evaluation Scenario}

Fig. 11 shows the resulting PDR and delay values for the considered protocols. In contrast to the previously analyzed UAV scenario, the vehicular scenario is more challenging for the routing protocols, since the larger network causes a higher number of potential routing paths from source to destination. Moreover, the physical dimensions of the scenario itself are larger and since the mobility characteristics of the vehicles are connectivity-unaware, connectivity is not always guaranteed. Therefore, the general PDR level is lower than in the UAV scenario. The highest PDR is achieved by the proposed predictive B.A.T.M.A.N. V, which exploits the high predictability of road-based vehicular mobility. Although the delay for AODV is on average more than five times larger than for the proactive protocols, all protocols fulfill the delay ETSI requirements for safety-related vehicular communication in the considered scenario [22]. 

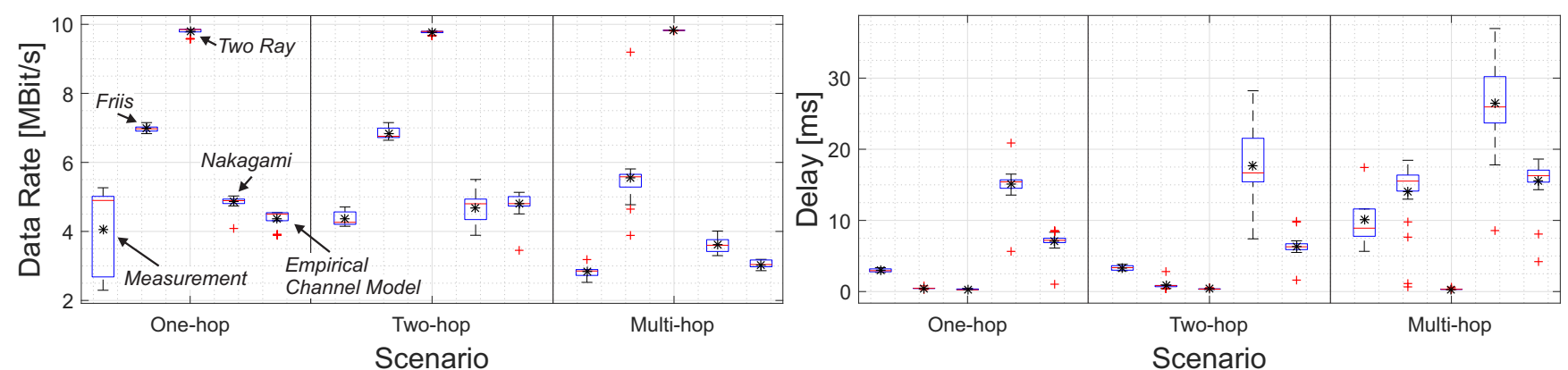

Fig. 6. Model validation: Comparison of measurements for data rate and delay with simulation results using different channel models.

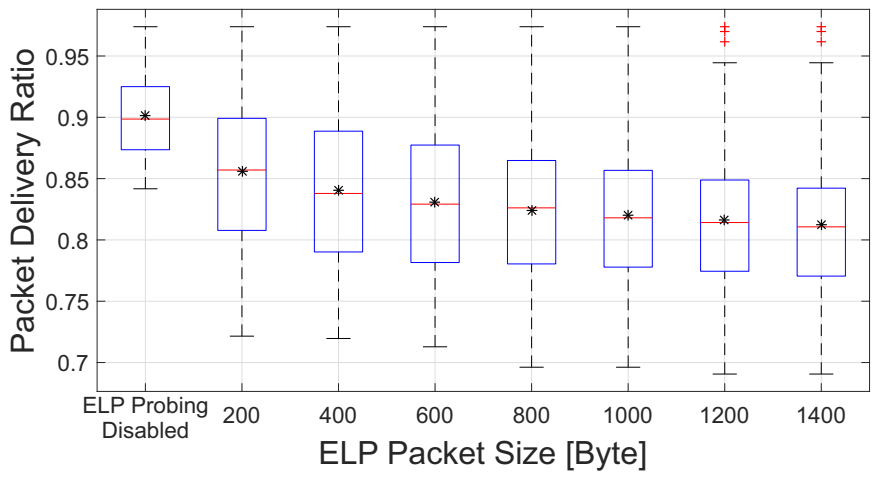

Fig. 7. Impact of ELP probing on the resulting data rate for different ELP packet sizes.

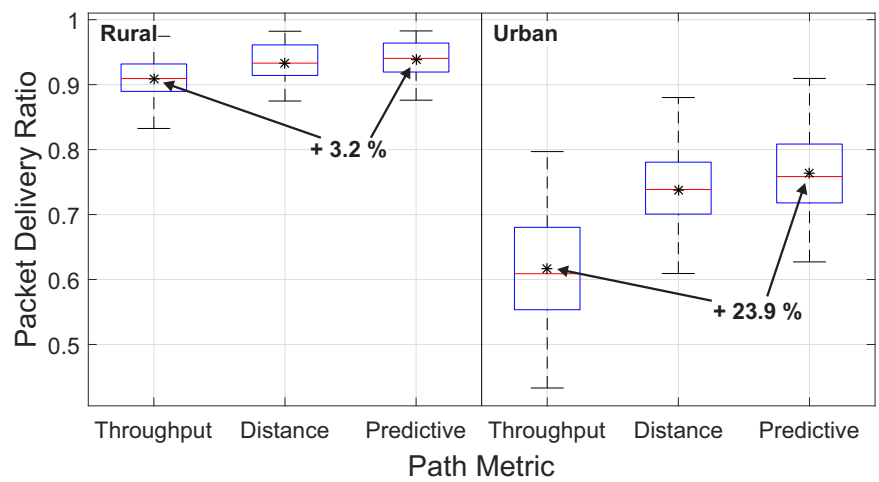

Fig. 8. Comparison of the native Throughput metric with the proposed Distance and Prediction methods for the rural and the urban evaluation scenario.

\section{CONCLUSION}

In this paper, we presented an analysis and optimization of the novel MAC layer routing protocol B.A.T.M.A.N. V for vehicular scenarios. In order to allow the simulative analysis, an open source simulation model was derived based on the Linux kernel implementation and validated using field measurements. Within comprehensive simulation studies, it was shown that some of the new features of B.A.T.M.A.N. V (e.g., ELP probing) decrease the routing efficiency in highly mobile sce- narios. In addition, our proposed novel routing metrics, which exploit distance-awareness and mobility prediction, showed a significantly better performance than the throughput estimation metric of B.A.T.M.A.N. V. In different case-studies for aerial and ground-based mobile ad-hoc networks, it was shown that the extended protocol is well-suited for maintaining connectivity within vehicular and UAV-based mesh networks and outperforms established topology-based routing approaches. In future work, we will exploit the proposed open source simulation model as a platform for optimizing vehicular mesh routing. A promising research topic is the determination of routing metrics based on reinforcement learning. Additionally, we will analyze the performance of using B.A.T.M.A.N. V in scenarios with heterogeneous communication technologies.

\section{ACKNOWLEDGMENT}

Part of the work on this paper has been supported by Deutsche Forschungsgemeinschaft (DFG) within the Collaborative Research Center SFB 876 "Providing Information by Resource-Constrained Analysis", project B4.

\section{REFERENCES}

[1] N. Bui, M. Cesana, S. A. Hosseini, Q. Liao, I. Malanchini, and J. Widmer, "A survey of anticipatory mobile networking: Context-based classification, prediction methodologies, and optimization techniques," IEEE Communications Surveys \& Tutorials, 2017.

[2] B. Sliwa, T. Liebig, R. Falkenberg, J. Pillmann, and C. Wietfeld, "Efficient machine-type communication using multi-metric contextawareness for cars used as mobile sensors in upcoming 5G networks," in 2018 IEEE 87th Vehicular Technology Conference (VTC-Spring), Porto, Portugal, Jun 2018, Best Student Paper Award.

[3] B. Sliwa, D. Behnke, C. Ide, and C. Wietfeld, "B.A.T.Mobile: Leveraging mobility control knowledge for efficient routing in mobile robotic networks," in IEEE GLOBECOM 2016 Workshop on Wireless Networking, Control and Positioning of Unmanned Autonomous Vehicles (WiUAV), Washington D.C., USA, Dec 2016.

[4] D. Johnson, N. Ntlatlapa, and C. Aichele, "A simple pragmatic approach to mesh routing using batman," in In 2nd IFIP International Symposium on Wireless Communications and Information Technology in Developing Countries, Pretoria, South Africa, 2008.

[5] T. Hardes, F. Dressler, and C. Sommer, "Simulating a city-scale community network: From models to first improvements for Freifunk," in 2017 International Conference on Networked Systems (NetSys), March 2017, pp. 1-7.

[6] A. Varga and R. Hornig, "An overview of the OMNeT++ simulation environment," in Proceedings of the 1st International Conference on Simulation Tools and Techniques for Communications, Networks and Systems \& Workshops, ser. Simutools '08. ICST, Brussels, Belgium, Belgium: ICST (Institute for Computer Sciences, Social-Informatics and Telecommunications Engineering), 2008, pp. 60:1-60:10. 

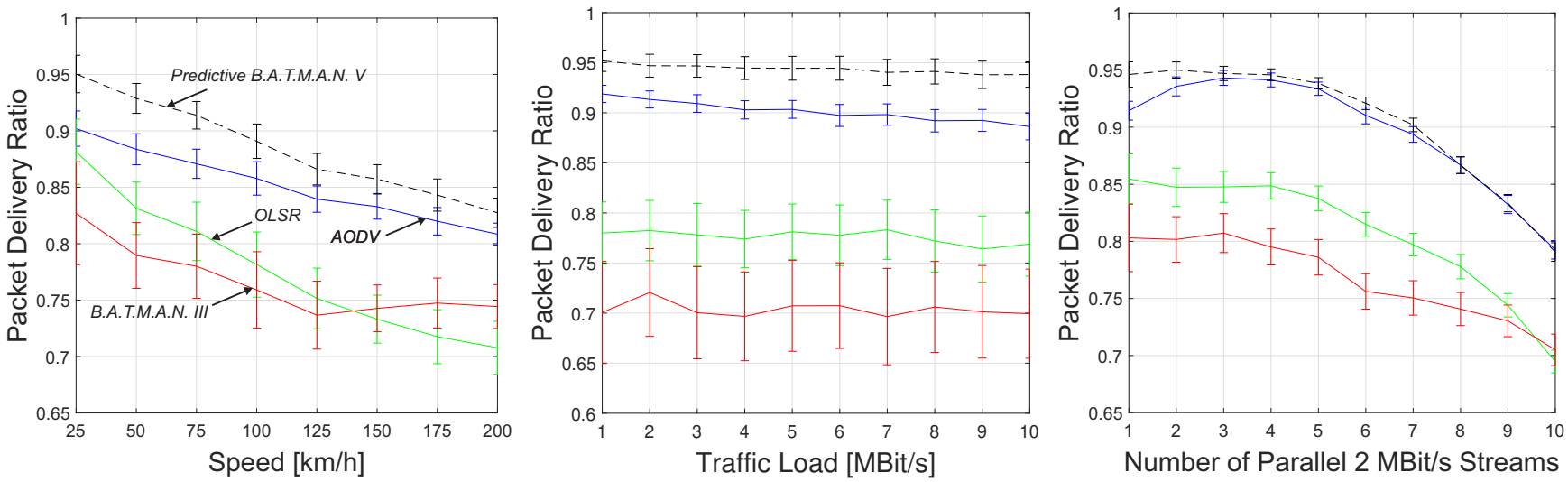

Fig. 9. Scalability analysis: Comparison of the resulting PDR with respect to vehicle speed, traffic load and number of streams for the proposed mobilitypredictive B.A.T.M.A.N. V and different established protocols (Rural channel).

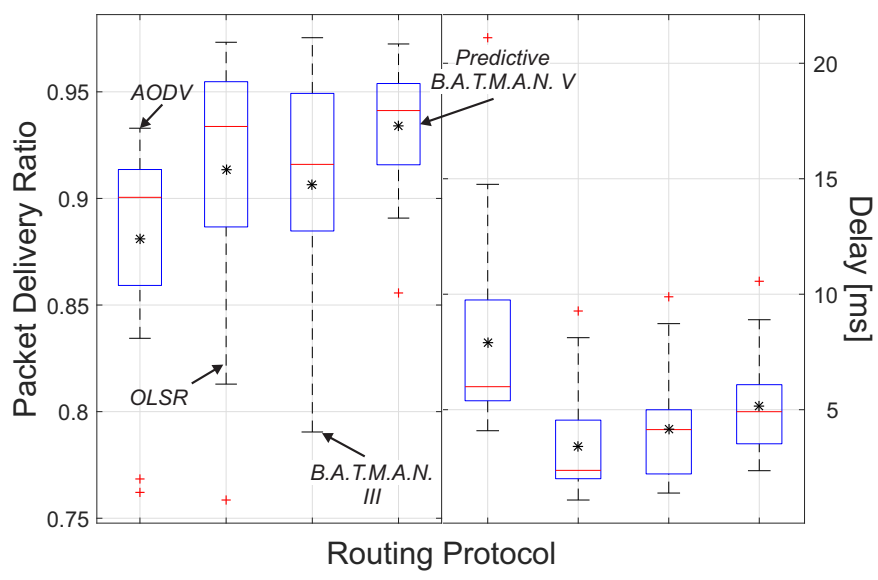

Fig. 10. Performance comparison using DDD mobility for UAV-based plume exploration (Urban channel, $10 \mathrm{UAVs}$ ).

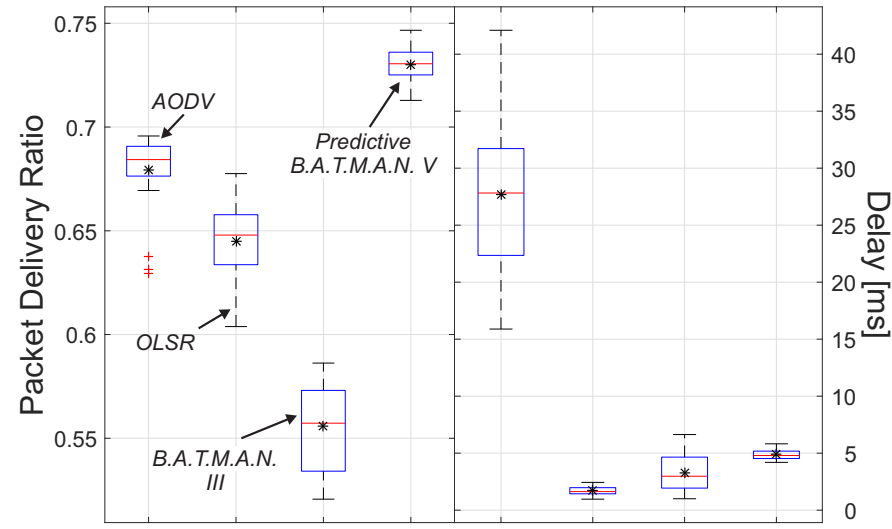

Fig. 11. Performance comparison for the vehicular network provisioning scenario (Urban channel, 30 vehicles).
[7] E. R. Cavalcanti, J. A. R. de Souza, M. A. Spohn, R. C. d. M. Gomes, and A. F. B. F. d. Costa, "VANETs' research over the past decade: Overview, credibility, and trends," SIGCOMM Comput. Commun. Rev., vol. 48, no. 2, pp. 31-39, May 2018.

[8] A. Awang, K. Husain, N. Kamel, and S. Aïssa, "Routing in vehicular ad-hoc networks: A survey on single- and cross-layer design techniques, and perspectives," IEEE Access, vol. 5, pp. 9497-9517, 2017.

[9] B. Karp and H. T. Kung, "GPSR: Greedy perimeter stateless routing for wireless networks," in Proceedings of the 6th Annual International Conference on Mobile Computing and Networking, ser. MobiCom '00. New York, NY, USA: ACM, 2000, pp. 243-254.

[10] G. Singal, V. Laxmi, M. S. Gaur, and V. Rao, "Moralism: Mobility prediction with link stability based multicast routing protocol in MANETs," Wireless Networks, vol. 23, no. 3, pp. 663-679, Apr 2017.

[11] H. Yang, M. Yu, and X. Zeng, "Link available time prediction based GPSR for vehicular ad hoc networks," in 2017 IEEE 14th International Conference on Networking, Sensing and Control (ICNSC), May 2017, pp. 293-298.

[12] C. E. Perkins and E. M. Royer, "Ad-hoc on-demand distance vector routing," in Mobile Computing Systems and Applications, 1999. Proceedings. WMCSA '99. Second IEEE Workshop on, Feb 1999, pp. 90100.

[13] X. Hu, J. Wang, and C. Wang, "Link stability prediction and its application to routing in mobile ad hoc networks," in 2009 2nd International Conference on Power Electronics and Intelligent Transportation System (PEITS), vol. 1, Dec 2009, pp. 141-144.

[14] H. Huang and S. Zhang, "A routing algorithm based on dynamic forecast of vehicle speed and position in VANET," International Journal of Distributed Sensor Networks, vol. 9, no. 6, p. 390795, 2013.

[15] H. Menouar, I. Guvenc, K. Akkaya, A. S. Uluagac, A. Kadri, and A. Tuncer, "UAV-enabled intelligent transportation systems for the smart city: Applications and challenges," IEEE Communications Magazine, vol. 55, no. 3, pp. 22-28, March 2017.

[16] T. Clausen and P. Jacquet, "Optimized link state routing protocol (OLSR)," RFC Editor, RFC 3626, October 2003.

[17] B. Sliwa, R. Falkenberg, and C. Wietfeld, "A simple scheme for distributed passive load balancing in mobile ad-hoc networks," in IEEE Vehicular Technology Conference (VTC-Spring), Sydney, Jun 2017.

[18] B. Sliwa, T. Liebig, R. Falkenberg, J. Pillmann, and C. Wietfeld, "Machine learning based context-predictive car-to-cloud communication using multi-layer connectivity maps for upcoming 5G networks," in 2018 IEEE 88th Vehicular Technology Conference (VTC-Fall), Chicago, USA, Aug 2018.

[19] B. Sliwa, "Simulation model and raw results," Oct 2018. [Online]. Available: http://doi.org/10.5281/zenodo.1466638

[20] B. Sliwa, J. Pillmann, F. Eckermann, L. Habel, M. Schreckenberg, and C. Wietfeld, "Lightweight joint simulation of vehicular mobility and communication with LIMoSim," in IEEE Vehicular Networking Conference (VNC), Torino, Italy, Nov 2017.

[21] D. Behnke, P. Bok, and C. Wietfeld, "UAV-based connectivity mainte- 
nance for borderline detection," in 2013 IEEE 77th Vehicular Technology Conference (VTC Spring), June 2013, pp. 1-6.

[22] ETSI, "Intelligent transport systems (ITS); vehicular communications; basic set of applications; definitions," ETSI TR 102638 V1.1.1 (200906), Tech. Rep., 2009. 